\begin{tabular}{|c|c|}
\hline Title & Comprehensive lipidomic profiling in serum and multiple tissues from a mouse model of diabetes \\
\hline Author(s) & $\begin{array}{l}\text { Chen, Zhen; Liang, Qiangrong; Wu, Y ue; Gao, Zijun; Kobay ashi, Satoru; Patel, Joy; Li, Cai rong; Cai, Fei; Zhang, } \\
\text { Y ouhua; Liang, Chongsheng; Chiba, Hitoshi; Hui, Shu-Ping }\end{array}$ \\
\hline Citation & $\begin{array}{l}\text { Metabolomics, 16(11), } 115 \\
\text { https://doi.org/10.1007/s11306-020-01732-9 }\end{array}$ \\
\hline Issue Date & $2020-10-16$ \\
\hline Doc URL & http:/hdl.handle.net/2115/82948 \\
\hline Rights & $\begin{array}{l}\text { This is a post-peer-review, pre copy edit version of an article published in Metabolomics. The final authenticated } \\
\text { version is available online at: https://doi.org/10.1007/\$11306-020-01732-9 }\end{array}$ \\
\hline Type & article (author version) \\
\hline Additional Information & There are other files related to this item in HUSCAP. Check the above URL. \\
\hline File Information & Hui2020.pdf \\
\hline
\end{tabular}

Instructions for use 


\section{Comprehensive Lipidomic Profiling in Serum and Multiple Tissues from a Mouse Model of Diabetes}

Zhen Chen ${ }^{1 \#}$, Qiangrong Liang ${ }^{2 \#}$, Yue $\mathrm{Wu}^{1}, \mathrm{Zijun}_{\mathrm{Gao}}{ }^{1}$, Satoru Kobayashi ${ }^{2}$, Joy Patel ${ }^{2}$, Cairong $\mathrm{Li}^{3}$, Fei $\mathrm{Cai}^{4}$, Youhua Zhang ${ }^{2}$, Chongsheng Liang ${ }^{1}$, Hitoshi Chiba ${ }^{5}$, Shu-Ping Hui ${ }^{*}$

1. Faculty of Health Sciences, Hokkaido University, Kita-12, Nishi-5, Kita-ku, Sapporo 060-0812, Japan

2. Department of Biomedical Sciences, New York Institute of Technology College of Osteopathic Medicine, Old Westbury, NY 11548, USA

3. Clinical Medical College, Hubei University of Science and Technology, Xianning 437100, China

4. Hubei Key Laboratory of Diabetes and Angiopathy, Hubei University of Science and Technology, Xianning 437100, China

5. Department of Nutrition, Sapporo University of Health Sciences, Nakanuma Nishi-4-2-1-15, Higashi, Sapporo 007-0894, Japan

* Corresponding author. E-mail: keino@hs.hokudai.ac.jp; Tel. : +81-11-706-3693

\# Zhen Chen and Qiangrong Liang contributed equally. 


\section{Abstract}

Introduction Diabetes mellitus is a serious metabolic disorder causing multiple organ damage in human. However, the lipidomic profiles in different organs and their associations are rarely studied in either diabetic patients or animals.

Objectives To evaluate and compare the characteristics of lipid species in serum and multiple tissues in a diabetic mouse model.

Methods Semi-quantitative profiling analyses of intact and oxidized lipids were performed in serum and multiple tissues from a diabetic mouse model fed a high fat diet and treated with streptozotocin by using LC/HRMS and MS/MS. The total content of each lipid class, and the tissue-specific lipid species in all tissue samples were determined and compared by multivariate analyses.

Results The diabetic mouse model displayed characteristic differences in serum and multiple organs: the brain and heart showed the largest reduction in cardiolipin, while the kidney had the most remarkable alterations in triacylglycerol. Interestingly, the lipidomic differences also existed between different regions of the same organ: triacylglycerol species with shorter fatty acyl chains decreased in renal medulla but increased in cortex; cardiolipin species with highly polyunsaturated fatty acyls decreased only in atrium but not in ventricle. Importantly, diabetes caused an accumulation of lipid hydroperoxides, suggesting that oxidative stress was induced in all organs except for the brain during the development of diabetes.

Conclusion These findings provided novel insight into the organ-specific relationship between diabetes and lipid metabolism, which might be useful for evaluating not only diabetic tissue injury but also the effectiveness of diabetic treatments.

\section{Keywords}

Lipidomics, type 2 diabetes mellitus, mouse model, multiple tissues, lipid hydroperoxides, oxidative stress 


\section{Introduction}

Diabetes mellitus has become a serious metabolic disorder throughout the world, representing one of the leading causes of morbidity and mortality (Bertoni et al. 2002), in which the type 2 diabetes mellitus accounts for approximately $90 \%$ of all the cases (International Diabetes Federation 2017). Diabetes not only leads to blood glucose imbalance, but also results in a series of whole-body complications, such as diabetic peripheral neuropathy, heart failure, and chronic kidney disease (CKD). These complications are becoming a major cause of increased mortality in diabetic patients (Gómez-Marcos et al. 2010; Klein and Waxman 2003; Papatheodorou et al. 2015). Therefore, it is imperative to characterize and understand diabetes-induced changes in different organs and tissues so that targeted therapies could be designed to reduce diabetic complications and mortality. Also, the potential metabolite markers could be used as screening tools for predicting the risk of these complications.

Oxidative stress is known to play a key role in the pathogenesis of diabetes and its complications (Giugliano et al. 1996). The increased reactive oxygen species (ROS) can attack biomolecules such as DNA, proteins, and lipids, damage cellular organelles including mitochondria and lipid droplets (Chen et al. 2017; Tsukui et al. 2019), and attenuate insulin secretion leading to diabetes (Evans et al. 2002). Although diabetic complications are associated with elevated levels of the lipid peroxidation products, most previous studies have focused on the oxidation end-products, e.g. thiobarbituric acid reactive substances, malondialdehydes, advanced glycation end products, and isoprostanes (Davì et al. 2005; Fatani et al. 2016; Greifenhagen et al. 2016). In our recent study on oxidized lipids, phospholipid hydroperoxides levels were found markedly elevated in the plasma from type 2 zebrafish model of diabetes (Chen et al. 2018). Besides the blood sample, Mora-Ortiz et al. investigated the metabolic biomarkers glucose and glutamate in kidney, spleen, eye, and other organs from type 2 diabetic $d b / d b$ mice (Mora-Ortiz et al. 2019). Nevertheless, comparative lipidomic studies on blood and organs from other diabetic animal models are still limited.

The aims of this study were to identify general or tissue specific lipid biomarkers in a mouse model of type 2 diabetes, and to clarify the relationship between type 2 diabetic status of the mice and their lipidomic profiling. To this end, we performed a comprehensive investigation of the intact lipids in serum and multiple tissues from mice with type 2 diabetes, and conducted multivariable statistics to discover the lipid species that differed in the diabetic mouse model. Furthermore, we characterized the intact lipids and lipid hydroperoxides that were linked 
to oxidative stress-induced damage in serum and different tissues.

\section{Materials and Methods}

\subsection{Chemicals}

Spectral grade solvents for lipid extraction and LC/MS analysis were obtained from Sigma-Aldrich (St. Louis, MO). The authentic internal standards (IS) for lipidomic analysis were purchased from Sigma-Aldrich and Avanti Polar Lipids (Alabaster, AL), including triglyceride (TG) 11:0/11:0/11:0, phosphatidylcholine (PC) 13:0/13:0, lysophosphatidylcholine (LPC) 15:0, phosphatidylethanolamine (PE) 15:0/15:0, lysophosphatidylethanolamine (LPE) 13:0, phosphatidylinositol (PI) 8:0/8:0, lysophosphatidylinositol (LPI) 13:0, and free fatty acid (FFA) 17:0. While cardiolipin (CL) (15:0) 4 was synthesized according to Ahmad et al. (2005). The mixed solution of all the IS was newly prepared with methanol (containing $0.006 \%$ BHT, w/v) and stored at $-80{ }^{\circ} \mathrm{C}$ until use. Other chemicals and reagents were of analytical grade unless specified.

\subsection{Induction of diabetes and preparation of samples}

All animal protocols conformed to the Public Health Service Guide for Care and Use of Laboratory Animals, which were approved by New York Institute of Technology College of Osteopathic Medicine (NYITCOM, Approval No. 2016-QL-01). The type 2 diabetes in FVB/N mice was induced as described previously with some modifications (Yu et al. 2017). The detailed information of animal feeding and modeling was described in Supplementary Material 1. Blood glucose levels were determined using a TRUEtrack glucometer (NIPRO DIAGNOSTICS, Fort Lauderdale, FL), and a fasting blood glucose level of $250 \mathrm{mg} / \mathrm{dL}$ or greater was considered diabetic. Echocardiography and hemodynamic measurement were examined as described in Supplementary Material 1. Mice of both control (Con) and high-fat diet/streptozotocin-induced diabetic (HFD-STZ) groups ( $\mathrm{n}=5$ for each) were anesthetized with $2 \%$ isoflurane and the blood was taken from the left ventricle into $3.5 \mathrm{~mL}$ Vacutainer Plus Plastic Serum Tubes (BD-367983-1, MedexSupply, Passaic, NJ 07055) for serum separation, and the separated serum samples were immediately stored in $-80^{\circ} \mathrm{C}$. Tissue samples were collected, including liver, brain, ventricle, atrium, renal medulla, renal cortex, and spleen, and immediately snap-frozen in liquid nitrogen and stored in $-80{ }^{\circ} \mathrm{C}$ until analysis.

\subsection{Lipid extraction from serum and tissues}

The lipid extraction procedure of serum samples was based on Bligh and Dyer (1959). In Brief, $100 \mu \mathrm{L}$ of serum $4 / 20$ 
sample was extracted with $800 \mu \mathrm{L}$ of cold chloroform/methanol 1:1 (v/v, with IS) twice, followed by drying under vacuum. For tissues, lipids were extracted by the modified method of Folch (1957). Briefly, approximately $10 \mathrm{mg}$ of tissue sample was weighed followed by adding $600 \mu \mathrm{L}$ of ice-cold chloroform/methanol 2:1 (v/v, with IS) and homogenized using a Bead Mill Homogenizer (Fisherbrand ${ }^{\circledR}$, Pittsburgh, PA). The extraction was performed twice for each sample, and the combined extract was dried under vacuum. Then, the dried lipids were dissolved in $100 \mu \mathrm{L}$ of methanol, centrifuged at $2500 \times g \quad{ }^{\circ} \mathrm{C}$ for $15 \min$ to remove any insoluble material, and thereafter stored at $-80^{\circ} \mathrm{C}$ until analysis. The sample pretreatment was completed within $1 \mathrm{~h}$ to avoid lipid auto-oxidation or degradation.

\subsection{LC/MS analysis}

Semi-quantitative lipidomics was performed with a Shimadzu Prominence HPLC system (Shimadzu Corp., Kyoto, Japan) and an LTQ Orbitrap mass spectrometer (Thermo-Fisher Scientific Inc., San Jose, CA) using electrospray ionization (ESI) mode in both positive and negative modes. The HPLC conditions, gradient elution programs, and MS detection parameters were set according to our previous work (Wu et al. 2019), described in Supplementary Material 1. To avoid bias and ensure the stability, samples were randomized in injection sequence, and the quality control (QC) samples which were prepared by pooling each serum sample before extraction, were inserted were tested at regular intervals throughout the sequence (Boretti et al. 2020). The raw data were processed by Xcalibur 2.2 (Thermo-Fisher Scientific Inc.) and SIEVE 2.0 (Thermo-Fisher Scientific Inc.). The identification of lipid molecules was executed with the help of LIPIDMAPS (Sud et al. 2007) as well as the comparison of standards synthesized in our laboratory previously (Hui et al. 2003, 2010). The amount of each lipid species was calculated by the IS amount and the peak area ratio of analyte/IS, as shown in

\section{Supplementary Material 2.}

\subsection{Statistics}

Data were expressed as means \pm standard deviations (SD). For pairwise comparisons, Shapiro-Wilk test for normality and nonparametric Mann-Whitney $U$-test were conducted using SPSS 26.0 (SPSS, Inc., Chicago, IL). While $Q$ values were calculated by the Benjamini-Hochberg false discovery rate correction (Benjamini and Hochberg 1995). For multivariate analysis of intact lipid species, principal component analysis (PCA) and hierarchical cluster analysis (HCA) with Ward's linkage method were conducted by JMP 14 pro (SAS Institute Inc., Cary, NC) without scaling or centering, while orthogonal partial least squares discriminant analysis $5 / 20$ 
(OPLS-DA) was processed using SIMCA-P 14.1 (Umetrics, Umeå, Sweden) with Pareto scaling of the semi-quantitative dataset and "Autofit" function to establish the optimal model for each tissue/serum. Correlation analysis was performed by calculating the Spearman correlation coefficient between the selected lipid species using SPSS 26.0. The heatmap was generated by the R package "heatmap3" (https://cran.r-project.org/package=heatmap3) with Z-score scaling. The lipidomic network was visualized by Cytoscape 3.7.1 (https://cytoscape.org/), in which lipid species and tissues/serum was set as nodes. The lipid variations (fold of change in HFD-STZ) were imported as connections of lipid-tissue/serum, while the HCA distance among lipid species were imported as connections of lipid-lipid (Shannon et al. 2003).

\section{Results and Discussion}

\subsection{Characterization of the diabetic mouse model}

The purpose of this study was to investigate the effects of overt diabetes on lipid metabolism and heart function. Type 2 diabetes accounts for more than $90 \%$ of all diabetic patients. Many previous studies have used HFD-fed animals to model type 2 diabetes. Unfortunately, HFD-feeding does not easily induce overt diabetes and the heart only shows very mild or no functional impairments. STZ diabetic animal model does have certain cardiac abnormalities, but it is type 1 diabetes which only accounts for $5 \%$ of all diabetic cases. Recent studies have used both HFD and low dose STZ (HFD-STZ) to induce a disease condition that more closely mimics overt type 2 diabetes than either HFD alone or STZ alone. This relatively newer HFD-STZ diabetic model has fast onset diabetes and clear cardiac functional impairment and has been increasingly used for investigating diabetic complications. Accordingly, our present study focused on the comparison between non-diabetic control and HFD-STZ-induced overt diabetes.

As shown in Table S1.1, blood glucose concentration was markedly higher in mice in HFD-STZ group than control (427 \pm 88 vs. $107 \pm 29 \mathrm{mg} / \mathrm{dL})$, indicating that HFD-STZ induced overt diabetes in mice. We determined their cardiac function with echocardiographic and hemodynamic measurements. The results showed that the diabetic mice had clearly impaired cardiac function as shown by a $14 \%$ decrease in ejection fraction, a $23 \%$ decrease in fractional shortening, an $11 \%$ reduction in left ventricle systolic pressure, a $20 \%$ reduction in $\mathrm{dP} / \mathrm{dt}-\mathrm{max}$, and an $18 \%$ reduction in $\mathrm{dP} / \mathrm{dt}-\mathrm{min}$. These results suggested that HFD-STZ-induced diabetes is a valid model for investigating diabetic complications especially diabetic cardiomyopathy.

$6 / 20$ 


\subsection{Comparison of different lipid classes in multiple organs}

The present LC/MS and MS/MS analysis identified a total of 173 intact lipid molecular species according to their retention behavior on HPLC as well as their protonated, ammoniated, or deprotonated ion peaks on HRMS (Table S1.2). Initially, the unsupervised statistic approach PCA using all the intact lipid species as variables was performed (Fig. 1). The first three principal components accounted for $22.5 \%, 16.4 \%, 13.9 \%$ of the total variation, respectively, indicating the complexity of the whole variance. The QC samples for evaluating the accuracy and robustness of the whole analytical procedures were clustered tightly in the graph, which confirmed the reliability of the data acquisition (Lee et al. 2013). The distinctive clustering of different organs was revealed, especially for liver, brain, ventricle, and atrium. Notably, serum samples grouped apart from other organs. Although organ samples are usually unavailable in clinic, the blood is widely used for diagnosis as well as metabolism studies, our results suggested the need to investigate multiple organs to acquire the entire lipid metabolic information.

The concentrations of individual lipid classes in serum and tissues are shown in Table 1. TG was the most abundant lipid class in liver, heart, kidney, and spleen, accounting for $51.6 \%$ of the total lipid content in the spleen as the highest, and $30.6 \%$ in the liver as the lowest. For the phospholipids, PE ranked the most abundant in all the investigated tissues, especially for brain, in which PE contributed $50.2 \%$ of the total lipids and $82.0 \%$ of the total phospholipids. These results were consistent with a previous study on phospholipids in rat brain which demonstrated PE as the major class (Choi et al. 2018), and the abundance of PE in brain might be related to the highly active interactions of membrane components (Söderberg et al. 1991). Additionally, PE in HFD-STZ group was lower than control in liver and atrium, but not in other tissues or sera. Apart from PE, total CL of HFD-STZ group also exhibited a depletion in all the tested tissues, especially in ventricle and atrium, which has been claimed to precipitate mitochondrial dysfunction in diabetic cardiomyopathy (Han et al. 2007). The lipid alterations in these tissues indicated lipid metabolic disorders, prompting us to explore the detailed characteristics of specific lipid species.

\subsection{Tissue-specific lipid species in the HFD-STZ diabetic mouse model}

To discover the most remarkable lipid metabolic impairments in the diabetic mouse model, we subsequently performed the supervised approach OPLS-DA for multiple tissues and sera individually, to recognize the specific modulations (Fig. S3.1-3.7). The explained variation $\left(R^{2} Y\right)$ and predicted variation $\left(Q^{2} Y\right)$ indicated that 
the resultant OPLS model was valid and highly reliable in each tissue/serum except for brain (Table S3.1). All the samples were clearly separated into two clusters in the scatter plots, suggesting distinct lipid profiles between control and the diabetic model. The top 10 increased or decreased lipid species in HFD-STZ groups were calculated according to their correlation $(\mathrm{p}(\mathrm{corr})[1])$, and the differences between the two groups were listed in Table S3.2.

It is interesting that, even within the same organ, different regions showed characteristic lipidomic profiles. For the heart (Fig. 2A-D), CL species with long chain and high unsaturation degree including 76:10, 76:11, 78:11, 78:12, and 78:13 were found as the top decreased lipids in the atrium from the HFD-STZ group, but only CL68:5 and CL72:8 were decreased in the ventricle. Moreover, the TG species with shorter fatty acyl chains (mainly 14:0, 16:0, and 16:1) were revealed as the top decreased lipids only in ventricle, while TG species enriched with 18:2 and 18:3 were elevated only in atrium. For kidney (Fig. 2E-H), the HFD-STZ medulla showed lower TG species with shorter fatty chains ( carbon number 42 to 48), but higher TGwith longer chains (carbon number 50 to 54). Whereas in cortex, though similar alteration was observed, both the decreased TG (carbon number 48 to 52$)$ and increased TG $(58: 7,58: 8$, and 62:12) showed even longer chain than medulla, indicating the specific metabolic roles of the lipidome in different regions of the same organ. Our results also supported the viewpoint that the alterations of fatty acyl composition in TG might suggest the differences in fat storage and lipid droplets. Besides, our findings exhibited a trend of CL immaturity in both medulla and cortex, suggesting a potential mitochondrial dysfunction in diabetic kidney (Chicco and Sparagna 2007).

CL species were also remarkably reduced in the brains (Table S4.1) of HFD-STZ mice (Fig. 3A). The percent reduction of the selected CL species $(P<0.05$ for all) ranged from $49.4 \%$ (CL76:8) to $87.2 \%$ (CL78:10) (Fig. 3B), suggesting a severe loss of mitochondrial function in the diabetic brain, which was associated with neuronal damage (Moreira et al. 2007). Moreover, studies on the brain mitochondria of diabetic rats showed that diabetes reduced the activities of the mitochondrial respiratory chain enzymes, leading to decreased ATP production and mitochondrial dysfunction (Mastrocola et al. 2005). In contrast, most of the increased lipid species in the brain contained linoleoyl, such as TG50:4 (16:1/18:2/16:1), PC40:8 (18:2/22:6), LPC18:2, and LPE18:2 (Fig. 3A and C). Long-chain polyunsaturated fatty acids (LCPUFAs) were reported as neuroprotective and anti-inflammatory constituents, including arachidonic acid, eicosapentaenoic acid, and docosahexaenoic acid (Das 2002, 1994), but the functional role of linoleic acid in the inflammatory response remains $8 / 20$ 
controversial (Fritsche 2008). Our results suggested that an imbalance of n-6 LCPUFAs might have adverse effect on the brain. Furthermore, we investigated linoleoyl-containing lipids to explore the variation of other related species (Fig. 2D). The predominant species PE38:6 (18:2/20:4), together with TG54:6 (18:1/18:3/18:2), TG54:7 (18:2/18:3/18:2), and all the CL species, showed a trend to decrease in HFD-STZ group, while the other linoleoyl-containing TG species and phospholipids, as well as FFA18:2, had the opposite trend. Considering that there was no difference in total linoleoyl amount in the brain, we suppose that it was fatty acyl-chain remodeling rather than fat accumulation that happened in the diabetic mouse brain. Given that tissue-specific glycerophospholipid remodeling was associated with diabetic complications (Sas et al. 2018), our data might provide further insight into the relationship between diabetes-induced disturbance of linoleoyl metabolism and the brain injury.

\subsection{Oxidized lipids in serum and organs from HFD-STZ diabetic mouse model}

Since oxidative stress plays a key role in the pathogenesis of diabetes and its complications, the oxidized lipids such as the lipid hydroperoxides were also determined, including the hydroperoxides for TG, PC, PE, and PI. The identification was based on their HRMS signals, the retention behavior on HPLC (Table S5.1), and the tandem MS fragmentations (Shrestha et al. 2019) (shown in Fig. S5.1, taking PCOOH34:2 (16:0/18:2-OOH) as an example). The amounts of them in control and HFD-STZ groups were listed in Supplementary Material 2. For serum, all hydroperoxides were markedly elevated, by 5.4-fold over control for TGOOH, 4.9-fold for PCOOH, 2.1-fold for PEOOH, and 1.8-fold for PIOOH in HFD-STZ group (Fig. 4A), which were consistent with our previous study on the zebrafish model of type 2 diabetes (Chen et al. 2018). Also, the elevation of PCOOH, a major product of phospholipid hydroperoxidation, agreed with the clinical study by Nagashima et al. on the serum from type 2 diabetic patients (Nagashima et al. 2002).

Furthermore, the levels of lipid hydroperoxide species varied depending on specific tissues (Fig. 4B-E). Most tissues exhibited increased levels of lipid hydroperoxides, including liver, heart (both ventricle and atrium), and kidney (both medulla and cortex). The livers from the HFD-STZ group showed an accumulation of total hydroperoxides for all lipid classes, with a 3.3-fold elevation of TGOOH, a 2.8-fold elevation of $\mathrm{PCOOH}$, 3.3-fold of PEOOH, and 2.7-fold of PIOOH. The HFD-STZ hearts showed the lowest accumulation of TGOOH compared with other tissues. Meanwhile, all the phospholipid hydroperoxides were increased in both ventricles and atria from the HFD-STZ group. Besides, lipid hydroperoxides showed no difference in the pattern

$$
9 / 20
$$


of alteration between ventricle and atrium. For kidney, the lipid hydroperoxide profile was very similar to that of the heart except that the TGOOH levels were increased in renal medulla from HFD-STZ group by 4.2 folds of control. These results suggested that metabolic syndrome and diabetes are strongly associated with oxidative stress(Hopps et al. 2010). Insulin resistance and inflammation increase ROS production, causing an imbalance between pro-oxidant and anti-oxidant species. The elevated levels of ROS were found in the liver of rats with high-fat diet induced metabolic syndrome (Auberval et al. 2014). Similarly, the increased ROS and lipid peroxidation were found in diabetic heart and kidney (Fukushima and Lopaschuk 2016; Mazumder et al. 2004; Thomas et al. 2015). In addition, renal medullary oxidative stress was involved in the pathogenesis of metabolic syndrome, diabetes, hypertension, and CKD (Makino et al. 2002; Mori et al. 2012; Palm et al. 2003). It was noted that the brain was the only organ that showed no differences in the levels of the lipid hydroperoxides from all lipid classes. This observation appeared to be incompatible with a previous study that implicated mitochondrial-derived ROS and insulin resistance in the pathogenesis of brain dysfunction in diabetes (Muriach et al. 2014). Also, our results showed a depletion of CL in diabetic brain, which could result in brain dysfunction. Therefore, the possible explanation of these results might be that, although oxidative stress and mitochondrial damage did occur in the brain, the lipid hydroperoxides were quickly purged by cellular degradation systems including the ubiquitin proteasome system (UPS) and autophagy. Nevertheless, the functional states of the UPS and autophagy in the diabetic brain remain to be determined.

$\mathrm{PCOOH}$ appeared to be the most abundant hydroperoxides that accumulated in other tissues. Therefore, to explore whether the associations between the accumulation of $\mathrm{PCOOH}$ and the alteration of other lipids were consistent among different tissues, we further selected PC (the intact form) together with LPC and FFA (the corresponding degradation products) to perform the correlation analysis, which were visualized as heatmaps with Spearman correlation coefficients for liver, ventricle, atrium, renal medulla, and renal cortex (Fig. 4F-J). In liver, PC species expressed unique correlating pattern: for those with the same carbon number, the more unsaturated PC species showed negative correlation with $\mathrm{PCOOH}$, while the less unsaturated ones showed positive correlation. This finding indicated that the balance of PC-composited fatty acyls in liver was tilted under the diabetic oxidative stress. Besides, the positive relation between PCOOH and most of LPC and FFA species suggested the oxidative stress-induced phospholipid degradation (Fig. 4F) (Chen et al. 2018; Ha et al. 2012). Heart and kidney exhibited opposite correlations in the majority of PC species: negative in the former, 
but positive in the latter, especially for atrium and renal cortex. Although the exact reason for this discrepancy remained unknown, it was suggested that the oxidation of PC, one of the major biological membrane components, might be through different mechanisms between the two organs. In terms of LPC, atrium, renal medulla and cortex shared a similar correlation pattern in which LPC18:2 and LPC20:5 were the only two species positively correlated to $\mathrm{PCOOH}$, whereas in ventricle the LPC species positively correlating to $\mathrm{PCOOH}$ were 18:0, 18:2, 20:4, and 22:6. The correlationship to FFA showed even more complicated patterns among these tissues. Nevertheless, all these results revealed that the oxidation of phospholipids (typically the formation of $\mathrm{PCOOH}$ ) is quite variable among multiple tissues, which requires not only lipidomics but also the integrative omics to identify the complicated regulating pathways.

To achieve a global view on all these lipidomic alterations, the dependency network was constructed between lipid species and serum/tissues (Fig. 5), in which the lines connecting lipid to serum/tissues illustrated the shifting trends in HFD-STZ group. Specifically, lipid hydroperoxides showed more clear positive connection to serum, liver, and kidney than intact lipids. CL exhibited the predominant decrease over other lipids in brain, heart, kidney, and spleen, while TG expressed varying levels depending on fatty acyl composition in atrium, renal medulla, and spleen. These findings showed that liver, atrium, and renal medulla provided large amounts of information regarding lipidomic alterations for understanding the HFD-STZ diabetic model and its complications. In addition, from the connection of each two lipid species (dependency as the distance calculated by HCA), CL species and phospholipid hydroperoxides were strongly associated, suggesting a possible relationship between mitochondrial dysfunction and membrane damage, which may help to predict mitochondria dysfunction in certain organs. In short, these comprehensive lipidomic data could help us better understand the molecular mechanisms underlying diabetes and its complications.

This study could be improved by more thoroughly characterizing the altered lipidomic profiles of multiple organs from animals with diabetes. One limitation of the present study is that the comparison was made only between the control and HFD-STZ mice, while the HFD-only and STZ-only mice were not included. This might have missed information important for understanding the underlying mechanisms that mediate the different phenotypes between these models, which clearly warrants further investigation in future studies. Additionally, as we focused on diabetic complications in major organs which cause patient death, adipose tissues were not investigated in this study, despite that they play an important role in the metabolic syndrome and diabetes. 
Besides, since the technique of MS imaging has been recently applied in tissue metabolomic changes, such as mediated albumin degradation in diabetic nephropathy (Grove et al. 2018) and renal oxidative stress in CKD (Hayasaka et al. 2016), this approach may be used to visualize lipid species in ventricle/atrium and medulla/cortex from diabetic model in the future.

\section{Conclusion}

In summary, type 2 diabetes-induced lipidomic changes in serum and multiple organs were characterized and compared between the control and HFD-STZ diabetic mice by using LC/HRMS and MS/MS. The lipidomic alterations even differed between ventricle and atrium as well as renal medulla and cortex, indicating the distinct lipid metabolic properties in different regions of the same organ. Moreover, diabetes caused increased accumulation of lipid hydroperoxides in all the organs examined except for the brain, suggesting that oxidative stress was induced in organs during the development of hyperlipidemia and diabetes. Together, these findings provided novel insight into the organ/tissue-specific relationship between diabetes and lipid metabolism. The specific lipidomic alterations might be used for not only the diagnosis of diabetic tissue injury but also the evaluation of the effectiveness for diabetic treatments.

\section{Acknowledgements}

This study was supported by (1) Japan Society for the Promotion of Science (JSPS) KAKENHI Grant Number 19K16531 to Zhen Chen, and (2) National Institutes of Health grant 1R15HL137130-01A1 to Qiangrong Liang.

\section{Author contributions}

Conceptualization: Shu-Ping Hui; Methodology: Zhen Chen; Formal analysis and investigation: Zhen Chen, Qiangrong Liang, Yue Wu, Zijun Gao, Chongsheng Liang; Writing - original draft preparation: Zhen Chen, Qiangrong Liang; Writing - review and editing: Hitoshi Chiba; Funding acquisition: Zhen Chen, Qiangrong Liang; Resources: Satoru Kobayashi, Joy Patel, Cairong Li, Fei Cai, Youhua Zhang; Supervision: Hitoshi Chiba, Shu-Ping Hui.

\section{Compliance with ethical standards}


Conflict of Interest: The authors declare that they have no conflict of interest.

Ethical approval: All procedures performed in studies involving animals were in accordance with the ethical standards of the Public Health Service Guide for Care and Use of Laboratory Animals in the New York Institute of Technology College of Osteopathic Medicine.

\section{References}

Ahmad, M., Ukkalam, M., \& Ahmad, I. (2005). Cardiolipin molecules and methods of synthesis. U.S., U.S.: NEOPHARM, INC.

Auberval, N., Dal, S., Bietiger, W., Pinget, M., Jeandidier, N., Maillard-Pedracini, E., et al. (2014). Metabolic and oxidative stress markers in Wistar rats after 2 months on a high-fat diet. Diabetology \& Metabolic Syndrome, 6(1), 130. https://doi.org/10.1186/1758-5996-6-130

Benjamini, Y., \& Hochberg, Y. (1995). Controlling the False Discovery Rate: A Practical and Powerful Approach to Multiple Testing. Journal of the Royal Statistical Society: Series B (Methodological), 57(1), 289-300. https://doi.org/10.1111/j.2517-6161.1995.tb02031.x

Bertoni, A. G., Krop, J. S., Anderson, G. F., \& Brancati, F. L. (2002). Diabetes-Related Morbidity and Mortality in a National Sample of U.S. Elders. Diabetes Care, 25(3), 471-475. https://doi.org/10.2337/diacare.25.3.471

Bligh, E. G., \& Dyer, W. J. (1959). A rapid method of total lipid extraction and purification. Canadian journal of biochemistry and physiology, 37(8), 911-7. https://doi.org/10.1139/o59-099

Boretti, F. S., Burla, B., Deuel, J., Gao, L., Wenk, M. R., Liesegang, A., \& Sieber-Ruckstuhl, N. S. (2020). Serum lipidome analysis of healthy beagle dogs receiving different diets. Metabolomics, 16(1), 1. https://doi.org/10.1007/s11306-019-1621-3

Chen, Z., Wu, Y., Ma, Y.-S., Kobayashi, Y., Zhao, Y.-Y., Miura, Y., et al. (2017). Profiling of cardiolipins and their hydroperoxides in HepG2 cells by LC/MS. Analytical and Bioanalytical Chemistry, 409(24), 5735-5745. https://doi.org/10.1007/s00216-017-0515-3

Chen, Z., Zang, L., Wu, Y., Nakayama, H., Shimada, Y., Shrestha, R., et al. (2018). Lipidomic Profiling on Oxidized Phospholipids in Type 2 Diabetes Mellitus Model Zebrafish. Analytical sciences, 34(10), 1201-1208. https://doi.org/10.2116/analsci.18P281

$13 / 20$ 
Chicco, A. J., \& Sparagna, G. C. (2007). Role of cardiolipin alterations in mitochondrial dysfunction and disease. American Journal of Physiology-Cell Physiology, 292(1), C33-C44. https://doi.org/10.1152/ajpcell.00243.2006

Choi, J., Yin, T., Shinozaki, K., Lampe, J. W., Stevens, J. F., Becker, L. B., \& Kim, J. (2018). Comprehensive analysis of phospholipids in the brain, heart, kidney, and liver: brain phospholipids are least enriched with polyunsaturated fatty acids. Molecular and Cellular Biochemistry, 442(1-2), 187-201. https://doi.org/10.1007/s11010-017-3203-x

Das, U. . (2002). Is type 2 diabetes mellitus a disorder of the brain? Nutrition, 18(7-8), 667-672. https://doi.org/10.1016/S0899-9007(02)00746-3

Das, U. N. (1994). Beneficial effect of eicosapentaenoic and docosahexaenoic acids in the management of systemic lupus erythematosus and its relationship to the cytokine network. Prostaglandins, Leukotrienes and Essential Fatty Acids, 51(3), 207-213. https://doi.org/10.1016/0952-3278(94)90136-8

Davì, G., Falco, A., \& Patrono, C. (2005). Lipid Peroxidation in Diabetes Mellitus. Antioxidants \& Redox Signaling, 7(1-2), 256-268. https://doi.org/10.1089/ars.2005.7.256

Evans, J. L., Goldfine, I. D., Maddux, B. A., \& Grodsky, G. M. (2002). Oxidative Stress and Stress-Activated Signaling Pathways: A Unifying Hypothesis of Type 2 Diabetes. Endocrine Reviews, 23(5), 599-622. https://doi.org/10.1210/er.2001-0039

Fatani, S. H., Babakr, A. T., NourEldin, E. M., \& Almarzouki, A. A. (2016). Lipid peroxidation is associated with poor control of type-2 diabetes mellitus. Diabetes \& Metabolic Syndrome: Clinical Research \& Reviews, 10(2), S64-S67. https://doi.org/10.1016/j.dsx.2016.01.028

Folch, J., Lees, M., Stanley, G. H. S., \& Sloane Stanley, G. H. (1957). A simple method for the isolation and purification of total lipides from animal tissues. The Journal of biological chemistry, 226(1), 497-509.

Fritsche, K. L. (2008). Too much linoleic acid promotes inflammation—doesn't it? Prostaglandins, Leukotrienes and Essential Fatty Acids, 79(3-5), 173-175. https://doi.org/10.1016/j.plefa.2008.09.019

Fukushima, A., \& Lopaschuk, G. D. (2016). Cardiac fatty acid oxidation in heart failure associated with obesity and diabetes. Biochimica et Biophysica Acta (BBA) - Molecular and Cell Biology of Lipids, 1861(10), 1525-1534. https://doi.org/10.1016/j.bbalip.2016.03.020

Giugliano, D., Ceriello, A., \& Paolisso, G. (1996). Oxidative stress and diabetic vascular complications. $14 / 20$ 
Diabetes care, 19(3), 257-67. https://doi.org/10.2337/diacare.19.3.257

Gómez-Marcos, M. A., Recio-Rodríguez, J. I., Rodríguez-Sánchez, E., Castaño-Sánchez, Y., de Cabo-Laso, A., Sánchez-Salgado, B., et al. (2010). Central blood pressure and pulse wave velocity: relationship to target organ damage and cardiovascular morbidity-mortality in diabetic patients or metabolic syndrome. An observational prospective study. LOD-DIABETES study protocol. BMC Public Health, 10(1), 143. https://doi.org/10.1186/1471-2458-10-143

Greifenhagen, U., Frolov, A., Blüher, M., \& Hoffmann, R. (2016). Site-specific analysis of advanced glycation end products in plasma proteins of type 2 diabetes mellitus patients. Analytical and Bioanalytical Chemistry, 408(20), 5557-5566. https://doi.org/10.1007/s00216-016-9651-4

Grove, K. J., Lareau, N. M., Voziyan, P. A., Zeng, F., Harris, R. C., Hudson, B. G., \& Caprioli, R. M. (2018). Imaging mass spectrometry reveals direct albumin fragmentation within the diabetic kidney. Kidney International, 94(2), 292-302. https://doi.org/10.1016/j.kint.2018.01.040

Ha, C. Y., Kim, J. Y., Paik, J. K., Kim, O. Y., Paik, Y.-H., Lee, E. J., \& Lee, J. H. (2012). The association of specific metabolites of lipid metabolism with markers of oxidative stress, inflammation and arterial stiffness in men with newly diagnosed type 2 diabetes. Clinical Endocrinology, 76(5), 674-682. https://doi.org/10.1111/j.1365-2265.2011.04244.x

Han, X., Yang, J., Yang, K., Zhongdan, Z., Abendschein, D. R., \& Gross, R. W. (2007). Alterations in myocardial cardiolipin content and composition occur at the very earliest stages of diabetes: A shotgun lipidomics study. Biochemistry, 46(21), 6417-6428. https://doi.org/10.1021/bi7004015

Hayasaka, T., Fuda, H., Hui, S.-P., \& Chiba, H. (2016). Imaging Mass Spectrometry Reveals a Decrease of Cardiolipin in the Kidney of NASH Model Mice. Analytical sciences, 32(4), 473-6. https://doi.org/10.2116/analsci.32.473

Hopps, E., Noto, D., Caimi, G., \& Averna, M. R. (2010). A novel component of the metabolic syndrome: The oxidative stress. Nutrition, Metabolism and Cardiovascular Diseases, 20(1), 72-77. https://doi.org/10.1016/j.numecd.2009.06.002

Hui, S.-P., Chiba, H., Jin, S., Nagasaka, H., \& Kurosawa, T. (2010). Analyses for phosphatidylcholine hydroperoxides by LC/MS. Journal of Chromatography B, 878(20), 1677-1682. https://doi.org/10.1016/j.jchromb.2010.04.011

$15 / 20$ 
Hui, S.-P., Murai, T., Yoshimura, T., Chiba, H., \& Kurosawa, T. (2003). Simple chemical syntheses of TAG monohydroperoxides. Lipids, 38(12), 1287-1292. https://doi.org/10.1007/s11745-003-1191-9

International Diabetes Federation. (2017). IDF Diabetes Atlas, 8th edn. Brussels, Belgium. International Diabetes Federation. http://www.diabetesatlas.org

Klein, J. P., \& Waxman, S. G. (2003). The brain in diabetes: molecular changes in neurons and their implications for end-organ damage. The Lancet Neurology, 2(9), 548-554. https://doi.org/10.1016/S1474-4422(03)00503-9

Lee, D.-K., Yoon, M. H., Kang, Y. P., Yu, J., Park, J. H., Lee, J., \& Kwon, S. W. (2013). Comparison of primary and secondary metabolites for suitability to discriminate the origins of Schisandra chinensis by GC/MS and LC/MS. Food Chemistry, 141(4), 3931-3937. https://doi.org/10.1016/j.foodchem.2013.06.064

Makino, A., Skelton, M. M., Zou, A., Roman, R. J., \& Cowley, A. W. (2002). Increased Renal Medullary Oxidative Stress Produces Hypertension. Hypertension, 39(2), 667-672. https://doi.org/10.1161/hy0202.103469

Mastrocola, R., Restivo, F., Vercellinatto, I., Danni, O., Brignardello, E., Aragno, M., \& Boccuzzi, G. (2005). Oxidative and nitrosative stress in brain mitochondria of diabetic rats. Journal of Endocrinology, 187(1), 37-44. https://doi.org/10.1677/joe.1.06269

Mazumder, P. K., O’Neill, B. T., Roberts, M. W., Buchanan, J., Yun, U. J., Cooksey, R. C., et al. (2004). Impaired Cardiac Efficiency and Increased Fatty Acid Oxidation in Insulin-Resistant ob/ob Mouse Hearts. Diabetes, 53(9), 2366-2374. https://doi.org/10.2337/diabetes.53.9.2366

Mora-Ortiz, M., Nuñez Ramos, P., Oregioni, A., \& Claus, S. P. (2019). NMR metabolomics identifies over 60 biomarkers associated with Type II Diabetes impairment in db/db mice. Metabolomics, 15(6), 1-16. https://doi.org/10.1007/s11306-019-1548-8

Moreira, P. I., Santos, M. S., Seiça, R., \& Oliveira, C. R. (2007). Brain mitochondrial dysfunction as a link between Alzheimer's disease and diabetes. Journal of the Neurological Sciences, 257(1-2), 206-214. https://doi.org/10.1016/j.jns.2007.01.017

Mori, T., Ogawa, S., Cowely, A. W., \& Ito, S. (2012). Role of renal medullary oxidative and/or carbonyl stress in salt-sensitive hypertension and diabetes. Clinical and Experimental Pharmacology and Physiology, 39(1), 125-131. https://doi.org/10.1111/j.1440-1681.2011.05653.x

$16 / 20$ 
Muriach, M., Flores-Bellver, M., Romero, F. J., \& Barcia, J. M. (2014). Diabetes and the Brain: Oxidative Stress, Inflammation, and Autophagy. Oxidative Medicine and Cellular Longevity, 2014, 1-9. https://doi.org/10.1155/2014/102158

Nagashima, T., Oikawa, S., Hirayama, Y., Tokita, Y., Sekikawa, A., Ishigaki, Y., et al. (2002). Increase of serum phosphatidylcholine hydroperoxide dependent on glycemic control in type 2 diabetic patients. Diabetes Research and Clinical Practice, 56(1), 19-25. https://doi.org/10.1016/S0168-8227(01)00353-9

Palm, F., Cederberg, J., Hansell, P., Liss, P., \& Carlsson, P.-O. (2003). Reactive oxygen species cause diabetes-induced decrease in renal oxygen tension. Diabetologia, 46(8), 1153-1160. https://doi.org/10.1007/s00125-003-1155-z

Papatheodorou, K., Banach, M., Edmonds, M., Papanas, N., \& Papazoglou, D. (2015). Complications of Diabetes. Journal of Diabetes Research, 2015(4), 1-5. https://doi.org/10.1155/2015/189525

Sas, K. M., Lin, J., Rajendiran, T. M., Soni, T., Nair, V., Hinder, L. M., et al. (2018). Shared and distinct lipid-lipid interactions in plasma and affected tissues in a diabetic mouse model. Journal of Lipid Research, 59(2), 173-183. https://doi.org/10.1194/jlr.M077222

Shannon, P., Markiel, A., Ozier, O., Baliga, N. S., Wang, J. T., Ramage, D., et al. (2003). Cytoscape: a software environment for integrated models of biomolecular interaction networks. Genome research, 13(11), 2498-504. https://doi.org/10.1101/gr.1239303

Shrestha, R., Chen, Z., Miura, Y., Yamamoto, Y., Sakurai, T., Chiba, H., \& Hui, S.-P. (2019). Identification of molecular species of phosphatidylcholine hydroperoxides in native and copper-oxidized triglyceride-rich lipoproteins in humans. Annals of Clinical Biochemistry: International Journal of Laboratory Medicine, 000456321988093. https://doi.org/10.1177/0004563219880932

Söderberg, M., Edlund, C., Kristensson, K., \& Dallner, G. (1991). Fatty acid composition of brain phospholipids in aging and in Alzheimer's disease. Lipids, 26(6), 421-425. https://doi.org/10.1007/BF02536067

Sud, M., Fahy, E., Cotter, D., Brown, A., Dennis, E. A., Glass, C. K., et al. (2007). LMSD: LIPID MAPS structure database. Nucleic Acids Research, 35(Database), D527-D532. https://doi.org/10.1093/nar/gk1838

Thomas, M. C., Brownlee, M., Susztak, K., Sharma, K., Jandeleit-Dahm, K. A. M., Zoungas, S., et al. (2015). Diabetic kidney disease. Nature Reviews Disease Primers, 1(1), 15018. https://doi.org/10.1038/nrdp.2015.18 
Tsukui, T., Chen, Z., Fuda, H., Furukawa, T., Oura, K., Sakurai, T., et al. (2019). Novel Fluorescence-Based Method To Characterize the Antioxidative Effects of Food Metabolites on Lipid Droplets in Cultured Hepatocytes. Journal of Agricultural and Food Chemistry, 67(35), 9934-9941. research-article. https://doi.org/10.1021/acs.jafc.9b02081

Wu, Y., Chen, Z., Darwish, W. S., Terada, K., Chiba, H., \& Hui, S.-P. (2019). Choline and Ethanolamine Plasmalogens Prevent Lead-Induced Cytotoxicity and Lipid Oxidation in HepG2 Cells. Journal of Agricultural and Food Chemistry, 67(27), 7716-7725. https://doi.org/10.1021/acs.jafc.9b02485

Yu, T., Sungelo, M., Goldberg, I., Wang, H., \& Eckel, R. (2017). Streptozotocin-Treated High Fat Fed Mice: A New Type 2 Diabetes Model Used to Study Canagliflozin-Induced Alterations in Lipids and Lipoproteins. Hormone and Metabolic Research, 49(05), 400-406. https://doi.org/10.1055/s-0042-110934 


\section{Figure Captions}

Fig. 1 Three-dimensional score plot by PCA revealed the lipidomic profiles of serum and different tissues. Samples are illustrated in different colors, and groups are indicated by different symbols: control (Con, *), high fat diet-STZ (HFD, $\square$ ), together with quality control samples (QCs, $\times$ ). The ellipses were illustrated manually for showing the clusters.

Fig. 2 Scatter plots of OPLS-DA for lipidomic characteristics in ventricle (A), atrium $(\mathbf{C})$, renal medulla (E), and renal cortex $(\mathbf{G})$ from control and HFD-STZ groups. (B, D, F, H) The top 10 increased/decreased lipid species in the corresponded tissues from HFD-STZ group, selected according to their correlation (p(corr)[1]). $Q$ values were calculated using Mann-Whitney $U$-test followed by Benjamini-Hochberg false discovery rate correction within all the selected lipid variables. The line in the middle of the box indicates the median value, the box indicates the first and third quartiles, while the whiskers indicate the minimum and the maximum values, respectively (there were no outliers).

Fig. 3 (A) Volcano plot of brain lipid profile differed between control and HFD-STZ groups, together with the selected lipid species which were decreased $(\mathbf{B})$ or increased $(\mathbf{C})$ in HFD-STZ group. $* P<0.05$, ** $P<0.01$, calculated using Mann-Whitney $U$-test. (D) Heatmap of the linoleoyl-contained lipid species in the brain from control and HFD-STZ groups. Semi-quantitative data was Z-score scaled, and lipid species was sorted by hierarchical cluster analysis with Ward's linkage method. The line in the middle of the box indicates the median value, the box indicates the first and third quartiles, while the whiskers indicate the minimum and the maximum values, respectively (there were no outliers).

Fig. 4 (A) Differences of lipid hydroperoxides between control (Con) and high fat diet-STZ (HFD) serum. (B-E) Comparison of lipid hydroperoxides in multiple tissues between Con and HFD groups, including TGOOH (B), PCOOH (C), PEOOH (D), and PIOOH (E). $P$ values were calculated using Mann-Whitney $U$-test, and $Q$ values were calculated by Benjamini-Hochberg false discovery rate correction within all the total lipids. (F-J) Correlation analysis of PCOOH species and the intact lipids PC, LPC, and FFA species in liver, ventricle, 
atrium, renal medulla, and cortex, calculated as the Spearman correlation coefficients. The line in the middle of the box indicates the median value, the box indicates the first and third quartiles, while the whiskers indicate the minimum and the maximum values, respectively (there were no outliers).

Fig. 5 Visualized connection of lipidomic alterations in diabetes model serum and tissues. Node shapes represent different types of variables ( $\odot$, lipid; $\square$, serum/tissue), the color and width of the lines connecting lipid to serum/tissue are proportional to the shifting importance, and the blue lines connecting two lipid species correspond to their high dependency (as the distance in hierarchical cluster analysis with Ward's linkage method). 


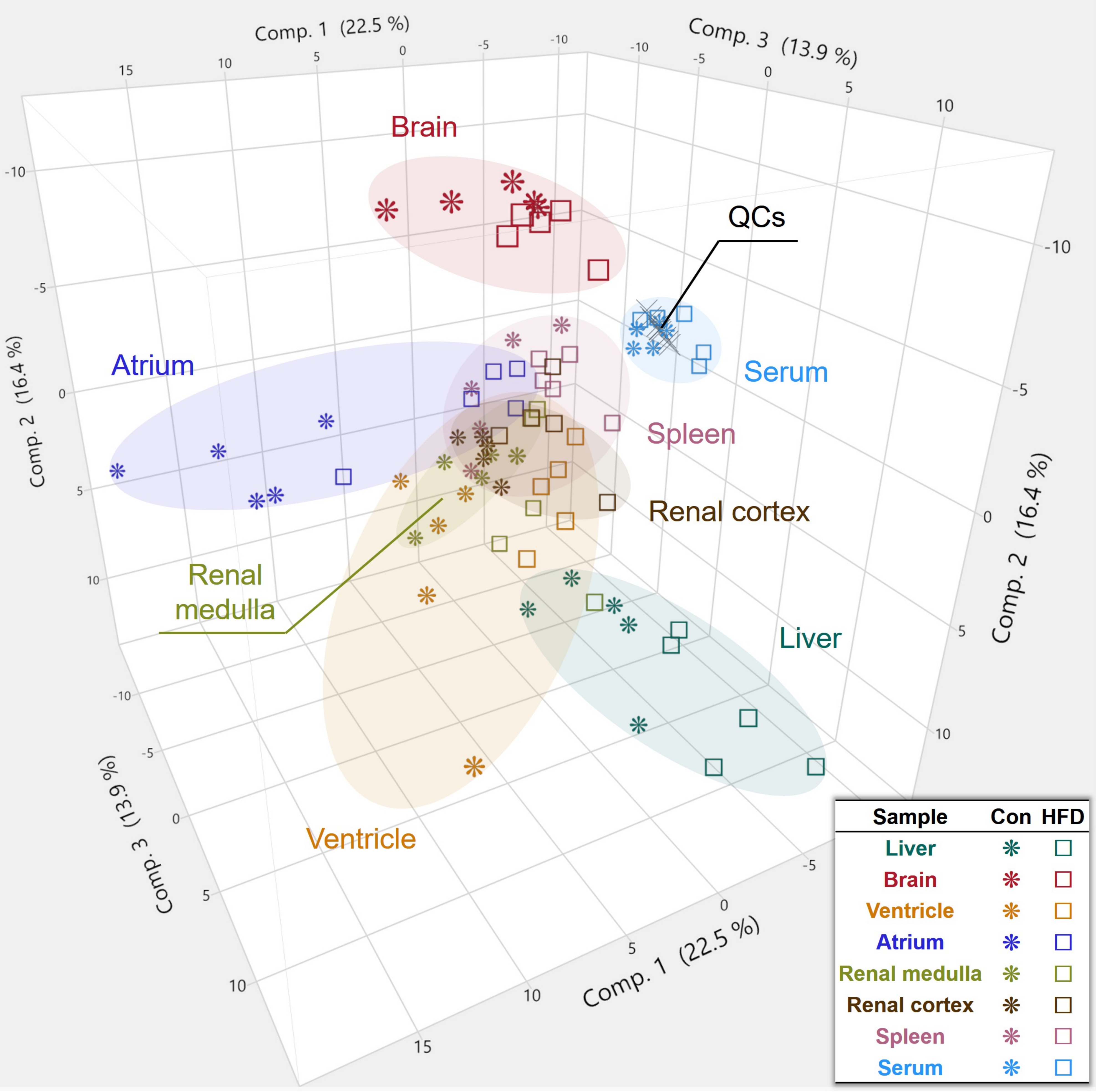




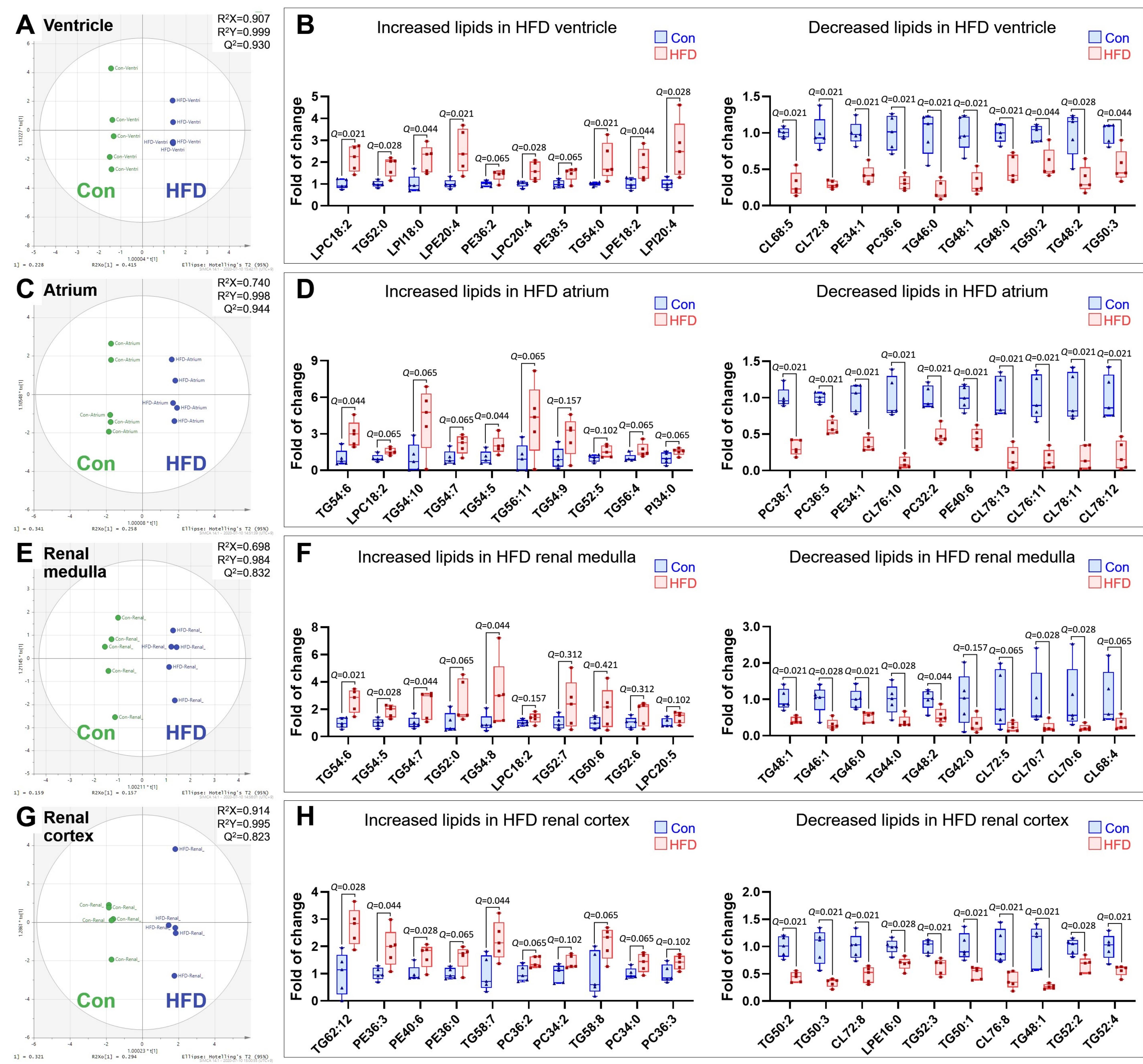



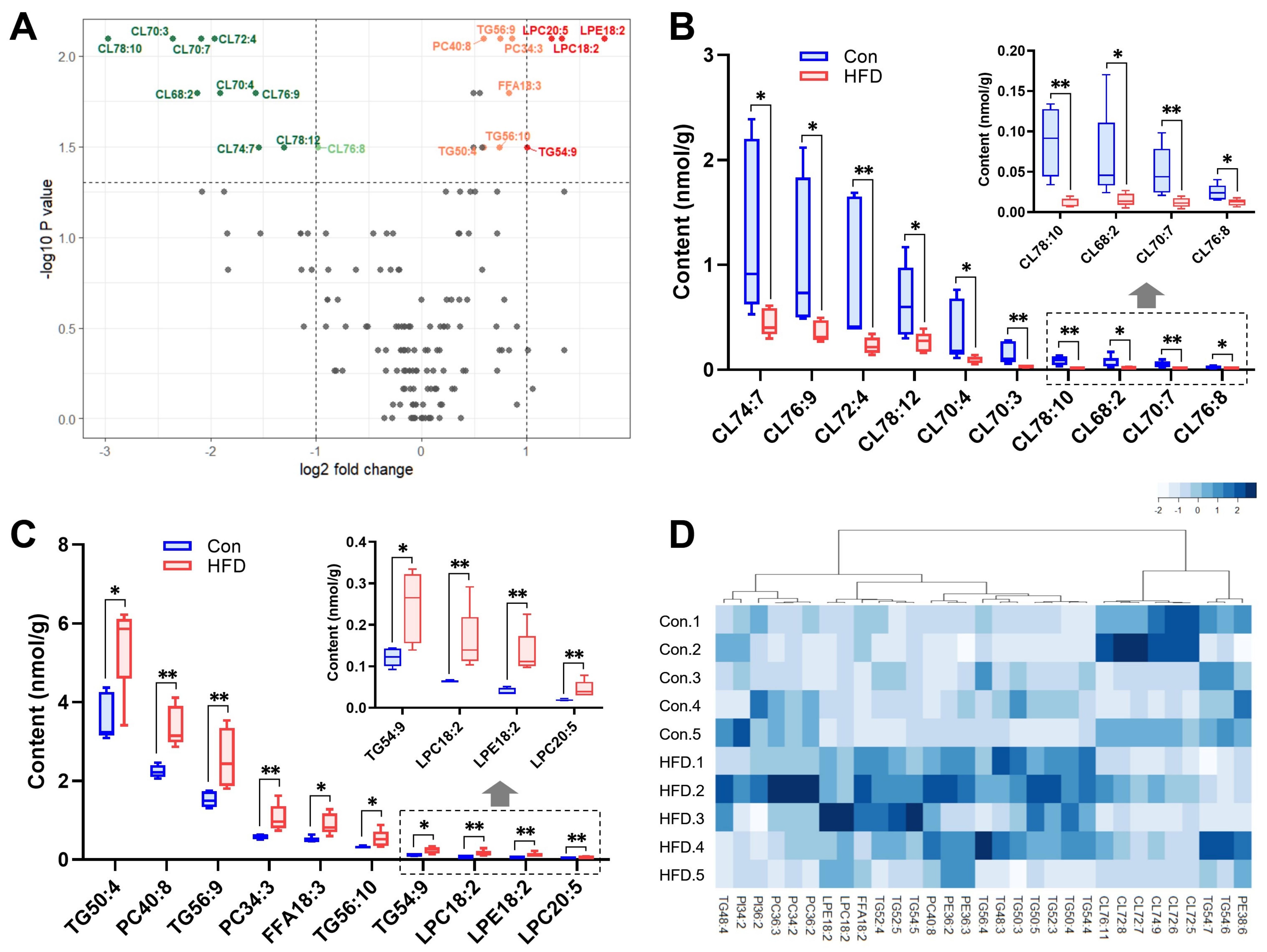


\section{A Total lipid-OOH concentration in serum}

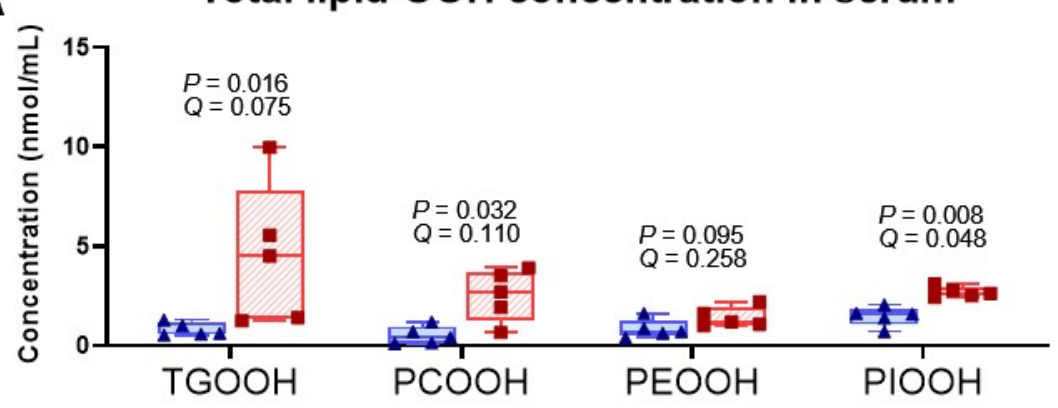

B

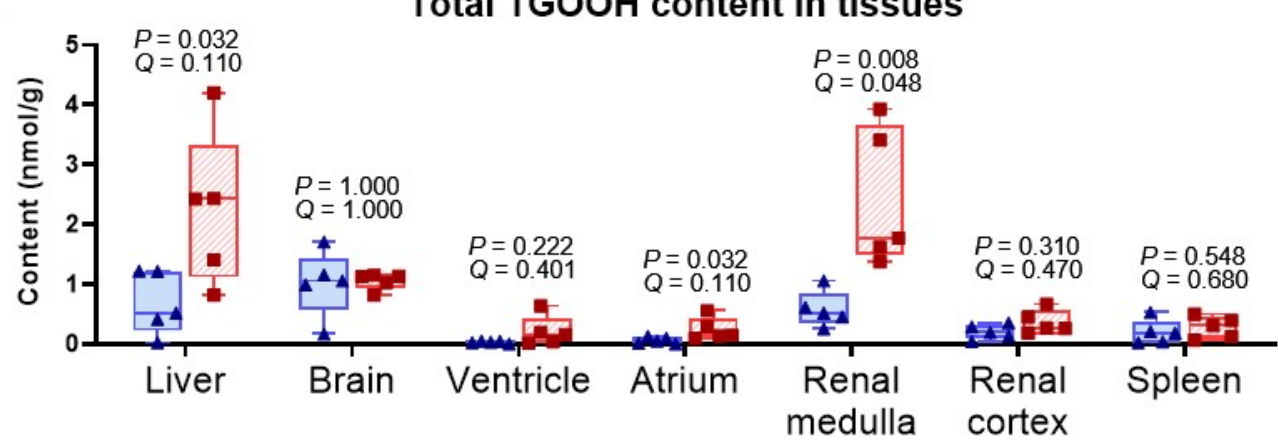

C

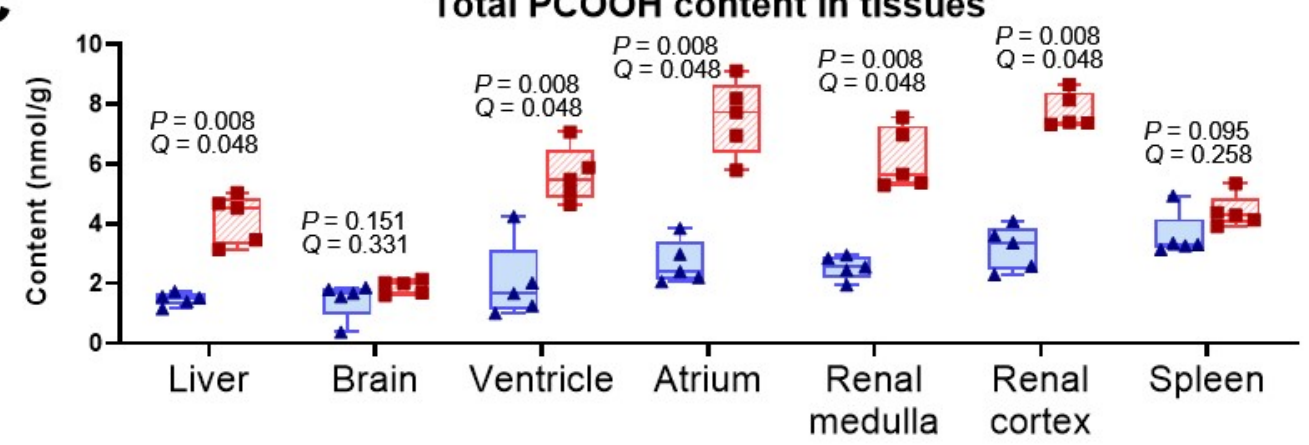

D

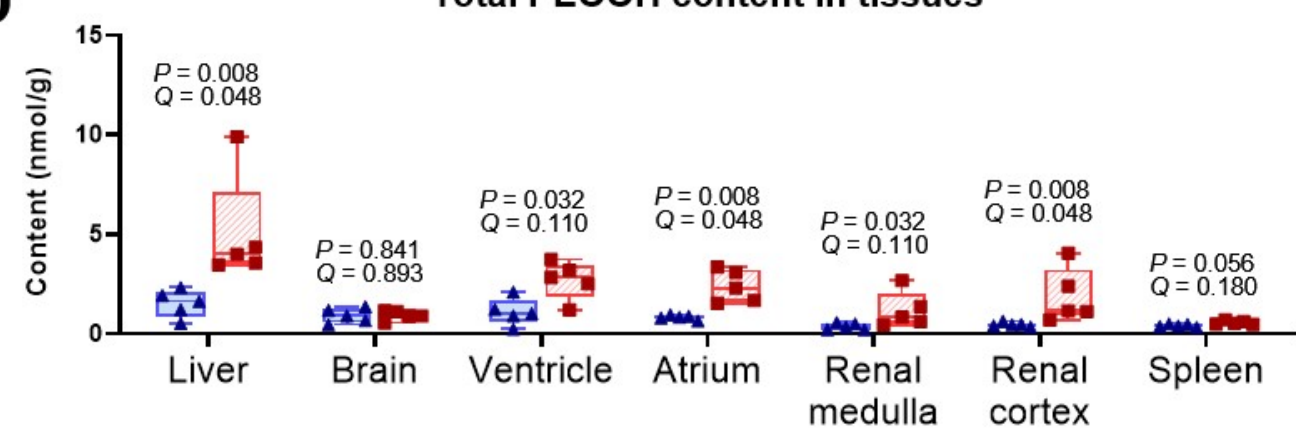

E

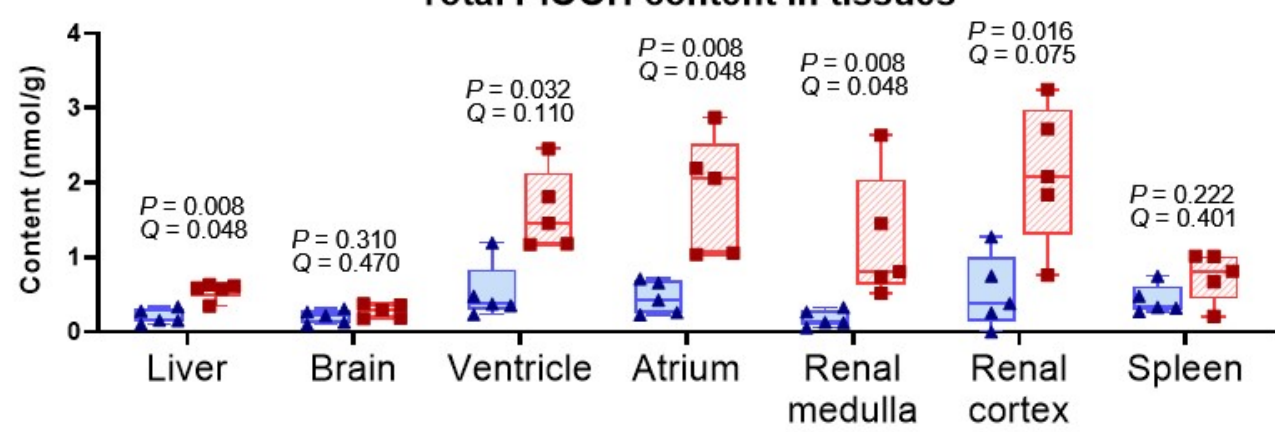

$\mathbf{F}$

Spearman Correlation in Liver

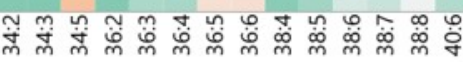
$\mathrm{PCOOH}$ species

G

Spearman Correlation in Ventricle

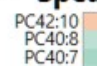

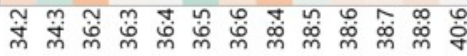
$\mathrm{PCOOH}$ species

I

Spearman Correlation in Renal Medulla SC42:10

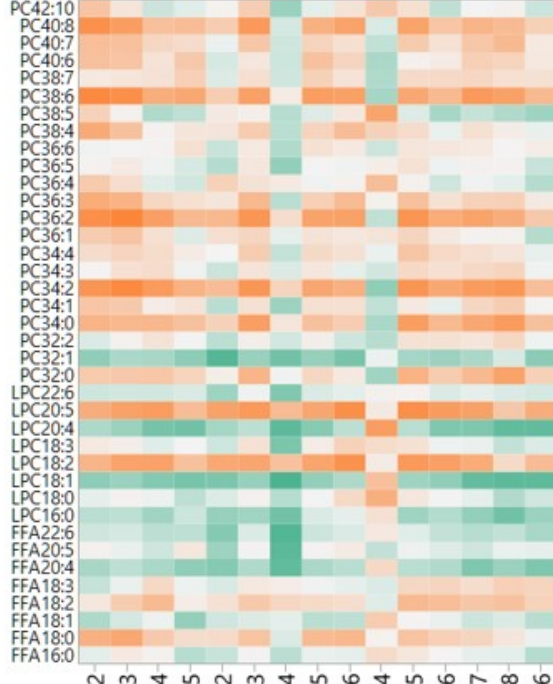

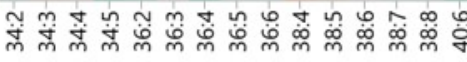
$\mathrm{PCOOH}$ species
H

Speraman Correlation in Atrium

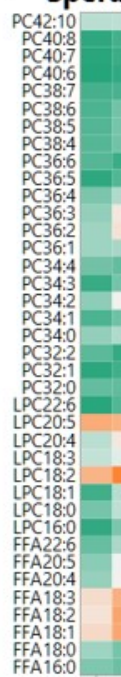

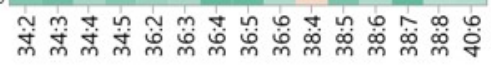
$\mathrm{PCOOH}$ species

$J_{s}$

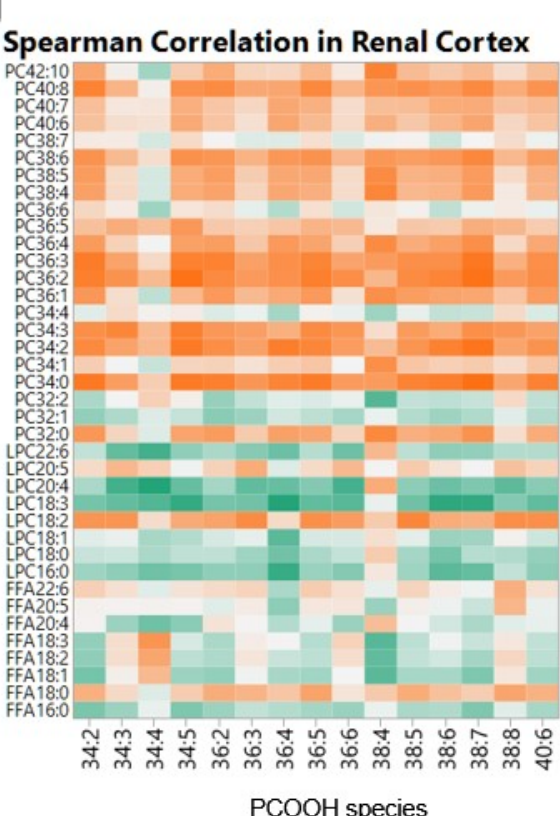


Table 1 Total intact lipid content in multiple tissues and serum, summarized by lipid classes $(\mathrm{n}=5)$

\begin{tabular}{|c|c|c|c|c|c|c|c|c|c|c|}
\hline \multirow{2}{*}{ Sample } & \multirow{2}{*}{ Group } & \multicolumn{9}{|c|}{ Total content for each class of intact lipids (nmol/g for tissues, or nmol/mL for serum) } \\
\hline & & $\mathrm{TG}$ & FFA & $\mathrm{PC}$ & LPC & $\mathrm{PE}$ & LPE & PI & LPI & $\mathrm{CL}$ \\
\hline \multirow{4}{*}{ Liver } & Con & $1720.8 \pm 315.1$ & $1101.2 \pm 239.7$ & $402.1 \pm 37.4$ & $88.8 \pm 26.4$ & $1951.8 \pm 284.6$ & $182.8 \pm 54.4$ & $172.1 \pm 20.9$ & $5.5 \pm 2.2$ & $6.4 \pm 3.8$ \\
\hline & HFD & $2037.9 \pm 419.7$ & $1301.2 \pm 241.5$ & $414.5 \pm 84.1$ & $112.4 \pm 33.0$ & $1315.5 \pm 41.3$ & $266.5 \pm 109.6$ & $163.0 \pm 19.9$ & $10.7 \pm 6.0$ & $4.2 \pm 3.1$ \\
\hline & $P$ value & 0.151 & 0.421 & 0.690 & 0.310 & $0.008 * *$ & 0.222 & 0.151 & 0.222 & 0.310 \\
\hline & $Q$ value & 0.331 & 0.571 & 0.799 & 0.470 & 0.048 & 0.401 & 0.331 & 0.401 & 0.470 \\
\hline \multirow{4}{*}{ Brain } & Con & $1234.9 \pm 92.0$ & $1181.9 \pm 135.3$ & $450.8 \pm 42.8$ & $17.0 \pm 2.3$ & $3132.7 \pm 540.7$ & $66.8 \pm 5.3$ & $136.5 \pm 14.9$ & $3.7 \pm 1.6$ & $11.2 \pm 7.0$ \\
\hline & HFD & $1321.3 \pm 207.9$ & $1185.5 \pm 198.3$ & $441.0 \pm 67.5$ & $20.8 \pm 6.5$ & $3101.2 \pm 545.2$ & $96.3 \pm 48.3$ & $117.6 \pm 18.0$ & $3.2 \pm 2.0$ & $5.1 \pm 1.6$ \\
\hline & $P$ value & 0.548 & 0.841 & 1.000 & 0.421 & 1.000 & 0.222 & 0.095 & 0.548 & 0.151 \\
\hline & $Q$ value & 0.680 & 0.893 & 1.000 & 0.571 & 1.000 & 0.401 & 0.258 & 0.680 & 0.331 \\
\hline \multirow{4}{*}{ Ventricle } & Con & $2414.3 \pm 398.2$ & $1383.6 \pm 287.3$ & $567.6 \pm 311.9$ & $41.0 \pm 5.2$ & $851.0 \pm 167.5$ & $75.1 \pm 20.1$ & $191.0 \pm 90.7$ & $1.0 \pm 0.3$ & $23.4 \pm 10.1$ \\
\hline & HFD & $1986.9 \pm 498.1$ & $1387.4 \pm 182.1$ & $325.2 \pm 76.2$ & $50.8 \pm 12.8$ & $820.7 \pm 135.2$ & $111.3 \pm 40.0$ & $145.3 \pm 29.3$ & $2.3 \pm 0.8$ & $9.8 \pm 2.9$ \\
\hline & $P$ value & 0.222 & 1.000 & 0.222 & 0.310 & 1.000 & 0.222 & 0.548 & $0.008 * *$ & $0.016 *$ \\
\hline & $Q$ value & 0.401 & 1.000 & 0.401 & 0.470 & 1.000 & 0.401 & 0.680 & 0.048 & 0.075 \\
\hline \multirow{4}{*}{ Atrium } & Con & $1785.7 \pm 589.0$ & $1086.4 \pm 106.0$ & $628.7 \pm 270.3$ & $57.1 \pm 17.3$ & $884.7 \pm 142.7$ & $73.5 \pm 42.2$ & $116.0 \pm 45.6$ & $1.0 \pm 0.8$ & $48.7 \pm 11.6$ \\
\hline & HFD & $1421.3 \pm 454.4$ & $1004.8 \pm 222.0$ & $304.5 \pm 91.5$ & $36.4 \pm 7.9$ & $656.2 \pm 78.2$ & $59.1 \pm 22.0$ & $118.2 \pm 11.2$ & $1.3 \pm 0.3$ & $19.6 \pm 12.8$ \\
\hline & $P$ value & 0.421 & 0.841 & 0.056 & 0.151 & $0.008 * *$ & 0.690 & 0.421 & 0.841 & $0.016 *$ \\
\hline & $Q$ value & 0.571 & 0.893 & 0.180 & 0.331 & 0.048 & 0.799 & 0.571 & 0.893 & 0.075 \\
\hline \multirow{4}{*}{$\begin{array}{l}\text { Renal } \\
\text { medulla }\end{array}$} & Con & $2697.3 \pm 390.5$ & $760.8 \pm 200.0$ & $388.8 \pm 113.1$ & $32.5 \pm 8.6$ & $1330.5 \pm 512.3$ & $76.6 \pm 22.3$ & $76.4 \pm 8.7$ & $3.2 \pm 0.9$ & $5.8 \pm 5.3$ \\
\hline & HFD & $2933.3 \pm 673.4$ & $686.0 \pm 180.6$ & $404.1 \pm 73.0$ & $27.1 \pm 7.4$ & $1204.2 \pm 433.9$ & $61.0 \pm 16.3$ & $83.0 \pm 17.9$ & $3.8 \pm 1.9$ & $2.4 \pm 0.8$ \\
\hline & $P$ value & 0.841 & 0.690 & 0.690 & 0.421 & 0.690 & 0.310 & 0.548 & 0.841 & 0.421 \\
\hline & $Q$ value & 0.893 & 0.799 & 0.799 & 0.571 & 0.799 & 0.470 & 0.680 & 0.893 & 0.571 \\
\hline \multirow{4}{*}{$\begin{array}{l}\text { Renal } \\
\text { cortex }\end{array}$} & Con & $2364.8 \pm 465.5$ & $599.2 \pm 57.3$ & $284.3 \pm 74.1$ & $40.2 \pm 9.2$ & $1945.3 \pm 286.3$ & $116.3 \pm 9.5$ & $70.2 \pm 15.1$ & $5.1 \pm 1.4$ & $10.6 \pm 3.9$ \\
\hline & HFD & $1591.0 \pm 294.4$ & $548.1 \pm 83.8$ & $345.3 \pm 81.3$ & $34.4 \pm 3.9$ & $2548.0 \pm 758.2$ & $87.5 \pm 14.8$ & $62.4 \pm 13.4$ & $4.3 \pm 1.4$ & $6.4 \pm 2.0$ \\
\hline & $P$ value & $0.016 *$ & 0.310 & 0.310 & 0.222 & 0.222 & $0.008 * *$ & 0.548 & 0.421 & 0.095 \\
\hline & $Q$ value & 0.075 & 0.470 & 0.470 & 0.401 & 0.401 & 0.048 & 0.680 & 0.571 & 0.258 \\
\hline
\end{tabular}

$1 / 2$ 


\begin{tabular}{|c|c|c|c|c|c|c|c|c|c|c|}
\hline \multirow{4}{*}{ Spleen } & Con & $1814.9 \pm 656.8$ & $571.0 \pm 111.7$ & $324.7 \pm 110.8$ & $28.3 \pm 4.5$ & $675.5 \pm 47.8$ & $52.2 \pm 2.1$ & $43.5 \pm 12.2$ & $1.8 \pm 0.3$ & $4.1 \pm 1.5$ \\
\hline & HFD & $1485.5 \pm 161.0$ & $770.6 \pm 189.0$ & $309.6 \pm 86.6$ & $38.6 \pm 15.2$ & $667.6 \pm 92.7$ & $48.5 \pm 7.1$ & $53.5 \pm 7.2$ & $2.1 \pm 1.1$ & $2.0 \pm 0.7$ \\
\hline & $P$ value & 0.421 & 0.095 & 0.841 & 0.151 & 0.548 & 0.151 & 0.310 & 0.690 & 0.032 \\
\hline & $Q$ value & 0.571 & 0.258 & 0.893 & 0.331 & 0.680 & 0.331 & 0.470 & 0.799 & 0.110 \\
\hline \multirow{4}{*}{ Serum } & Con & $884.0 \pm 111.0$ & $1170.1 \pm 280.2$ & $189.3 \pm 14.4$ & $33.0 \pm 4.6$ & $49.1 \pm 17.8$ & $45.9 \pm 12.3$ & $17.2 \pm 3.3$ & $10.3 \pm 1.5$ & Not detected \\
\hline & HFD & $1043.2 \pm 133.0$ & $1244.0 \pm 382.2$ & $214.3 \pm 40.7$ & $45.5 \pm 14.9$ & $50.4 \pm 15.6$ & $59.2 \pm 16.4$ & $19.6 \pm 3.7$ & $17.9 \pm 7.5$ & Not detected \\
\hline & $P$ value & 0.151 & 0.841 & 0.310 & 0.095 & 1.000 & 0.151 & 0.310 & 0.032 & - \\
\hline & $Q$ value & 0.331 & 0.893 & 0.470 & 0.258 & 1.000 & 0.331 & 0.470 & 0.110 & - \\
\hline
\end{tabular}

Con, control group; HFD, high fat diet-STZ group. Data were expressed as means \pm standard deviations. $* P<0.05, * * P<0.01$ vs control group, calculated using MannWhitney $U$-test. Q value was calculated by Benjamini-Hochberg false discovery rate (FDR) correction (FDR rate was set at 0.1 ) within all the total lipids.

Abbreviations of lipid classes: TG, triacylglycerol; FFA, free fatty acid; PC, phosphatidylcholine; LPC, lysophosphatidylcholine; PE, phosphatidylethanolamine; LPE, lysophosphatidylethanolamine; PI, phosphatidylinositol; LPI, lysophosphatidylinositol; CL, cardiolipin. 